\title{
Effect of Fermentation with Two Molds on Characteristics of Chicken Meat
}

\author{
Liping Guo, ${ }^{1,2}$ Yan Li, ${ }^{1}$ Shengchao Ding, ${ }^{1}$ Baowei Wang, ${ }^{1}$ Yinglian Zhu, ${ }^{1}$ Bin Pang, \\ Ming Huang, ${ }^{3}$ Harvey Ho, ${ }^{4}$ Jiying $Y u,{ }^{5}$ and Jingxin Sun $\mathbb{D}^{1,2}$ \\ ${ }^{1}$ College of Food Science and Engineering, Shandong Reseach Center for Meat Food Quality Control, \\ Qingdao Agricultural University, Qingdao 266109, China \\ ${ }^{2}$ Qingdao Special Food Research Institute, Qingdao 266109, China \\ ${ }^{3}$ National R\&D Branch Center for Poultry Meat Processing Tech., Nanjing Huangjiaoshou Food Sci. \& Tech. Co. Ltd., \\ Nanjing 211226, China \\ ${ }^{4}$ Bioengineering Institute, The University of Auckland, Auckland, New Zealand \\ ${ }^{5}$ Hainan (Tanniu) Wenchang Chicken Co. Ltd., Haikou 571133, Hainan, China
}

Correspondence should be addressed to Jingxin Sun; jxsunqau@126.com

Received 3 September 2020; Revised 4 December 2020; Accepted 30 January 2021; Published 10 February 2021

Academic Editor: Luis Patarata

Copyright (c) 2021 Liping Guo et al. This is an open access article distributed under the Creative Commons Attribution License, which permits unrestricted use, distribution, and reproduction in any medium, provided the original work is properly cited.

In the present study, we investigated the characteristics of chicken meat fermented with Penicillium nalgiovense and Penicillium chrysogenum. Hardness and springiness gradually decreased, while gumminess gradually increased during fermentation. Fermentation with $P$. chrysogenum led to higher hardness and lower gumminess than fermentation with $P$. nalgiovense. Fermentation with two molds resulted in similar microstructure, such as granule formation and fractured myofibril. The highest percentage of secondary structure was a-helix, and tyrosine residues were buried after fermentation. $P$. nalgiovense-fermented samples contained more bound water, lower relative content of alkanes, and higher relative content of aldehydes than $P$. chrysogenumfermented samples.

\section{Introduction}

Mold-fermented meat processing originated in Italy and concentrated especially in Southern European regions previously [1]. Nowadays, it has been spread around the world. In particular, Penicillium genera is the most widespread in meat products, probably because they can grow at lower temperatures and $a w$ (water activity) than other molds normally present in meat [2]. However, the colonization of meat surface by undesirable molds can lead to the deterioration of food quality, such as off flavors, colour of conidia, and floccose mycelium on the casing [3] and even produce highly toxic secondary metabolites with relevant toxic effects on consumer health [4]. In contrast, inoculation with appropriate molds in fermented meat can contribute to taste and aroma, improve the stabilisation of colour, and delay the rancidity [5].
To date, many nontoxigenic commercial molds have been used as starter culture for meat fermentation, including Penicillium aurantiogriseum, Penicillium camemberti, Penicillium roqueforti, Penicillium nalgiovense, and Penicillium chrysogenum. Previous published literature studies reported that inoculation with $P$. chrysogenum and $P$. camemberti increased the contents of free amino acids, free fatty acids, and volatile compounds as well as the overall quality of pork and beef products $[2,6,7]$. On the other hand, $P$. nalgiovense also has been used as a protective starter culture in processing of dry-fermented sausages [3, 8], which could avoid growth of toxigenic molds during the drying and fermenting processes [3]. Similarly, $P$. aurantiogriseum and $P$. roquefort $i$ could improve the sensory properties of products via increasing the content of flavor compounds [5, 9]. However, little information is available in the literature regarding the effect of molds on quality of fermented chicken meat. 
It has been reported that high consumption of processed red meat (ham, sausages, bacon, frankfurters, salami, etc.) increased the risk of several major chronic diseases (some carcinomas, atherosclerotic cardiovascular disease, type II diabetes, etc.) and preterm mortality $[10,11]$. Therefore, being a white meat, chicken meat is increasingly consumed due to its less fat and cholesterol, as well as a higher proportion of polyunsaturated fatty acids compared with red meat $[12,13]$. However, the kinds of processed chicken products are fewer than red meat. Moreover, little information is available about the influence of fermentation on characteristics of cooked chicken meat. Hence, the main objective of the present study was to compare the effects of fermentation with $P$. nalgiovense and $P$. chrysogenum on quality of chicken breast meat product, including texture, microstructure, protein structure, and volatile compounds.

\section{Materials and Methods}

2.1. Preparation of the Spore Suspension of Penicillium nalgiovense and Penicillium chrysogenum. The starter culture of $P$. nalgiovense and $P$. chrysogenum was purchased from the China Center of Industrial Culture Collection and saved at $4^{\circ} \mathrm{C}$ in Czapek's medium. The preparation of the spore suspension, treatment of chicken breast meat, and the fermenting and ripening conditions have been described in a previous study [14]. Finally, the working spore concentration was about $10^{6}$ spores $/ \mathrm{mL}$. Chicken meat manufacture was replicated three times.

2.2. Preparation of the Fermented Chicken Meats. The fresh chicken breast meat was purchased from a local farmers' market in Qingdao and was transported to the laboratory under iced conditions. They were cut into uniform size $(3 \mathrm{~cm} * 3 \mathrm{~cm} * 1 \mathrm{~cm})$ and 200 pieces in each group. Firstly, the chicken breast meat was sterilized with high-pressure steam $(0.1 \mathrm{MPa})$ at $121^{\circ} \mathrm{C}$ for $15 \mathrm{~min}$, then superficially inoculated with $2 \mathrm{~mL}$ of $P$. nalgiovense and $P$. chrysogenum, fermented at $27 \pm 1^{\circ} \mathrm{C}$ and $80 \pm 5 \%$ relative humidity for 7 days (primary fermentation). Afterwards, they were immersed in $4 \%$ sodium chloride solution and fermented at $30^{\circ} \mathrm{C}$ for 14 days (postfermentation). Each treatment was replicated three times.

2.3. Texture Analysis. A TA-XT2i texture analyzer (Stable Micro Systems, Surrey, UK) equipped with a cylindrical probe (P 0.25ss) was used to determine hardness, springiness, and gumminess at $25^{\circ} \mathrm{C}$. This procedure involved cutting samples approximately $3.0 \mathrm{~cm} \times 3.0 \mathrm{~cm} \times 1.0 \mathrm{~cm}$ (length $\times$ width $\times$ height). The texture analyzer conditions were set as follows: pretest speed $2.0 \mathrm{~mm} / \mathrm{s}$, test speed $1.0 \mathrm{~mm} / \mathrm{s}$, post-test speed $5.0 \mathrm{~mm} / \mathrm{s}$, compressed distance $8.0 \mathrm{~mm}$, and applied trigger force $5 \mathrm{~g}$. During primary fermentation, samples of 1,4 , and 7 days were used for analysis. During postfermentation, samples were analyzed every other day. Texture Expert version 1.22 software (Stable Micro System, Surrey, UK) was used to calculate the values of hardness, springiness, and gumminess. Each batch was analyzed six times; therefore, each value presented in this study is the mean of 18 data.

2.4. Microstructure Evaluation. Scanning electron microscope (SEM) and transmission electron microscopy (TEM) were used to view the microstructural changes of fermented chicken breast meat. For chicken meat, $5 \mathrm{~mm} \times 5 \mathrm{~mm} \times 3 \mathrm{~mm}$ (length $\times$ width $\times$ thickness) of samples were cut in a pool of fixing solution of $2 \%$ glutaraldehyde in $0.1 \mathrm{M}$ sodium phosphate buffer ( $\mathrm{pH} \mathrm{7.4)}$ at $4^{\circ} \mathrm{C}$ for $24 \mathrm{~h}$. They were then washed six times with the same buffer for a period of $20 \mathrm{~min}$. After that $1 \%$ osmium tetroxide in the same buffer was added for postfixation and kept at $4^{\circ} \mathrm{C}$ for $2 \mathrm{~h}$. Then, the samples were washed six times for 10 min each, using 0.1 M sodium phosphate buffer ( $\mathrm{pH} 7.4$ ). Samples were dehydrated in serial dilutions of ethanol (once in $50,60,70,80,90$, and three times in $100 \%$ ), each one for $15 \mathrm{~min}$. Afterwards, samples were prepared to be observed using a JSM-7500F scanning electron microscope (JEOL Ltd., Tokyo, Japan) with an accelerating voltage of $2.00 \mathrm{kV}$. For TEM examination, dehydrated samples were embedded in Epon-812, then stained with uranyl acetate and lead citrate, and observed with a HT7700 transmission electron microscope (Hitachi Ltd., Tokyo, Japan) at an operating voltage of $80 \mathrm{kV}$.

2.5. Raman Spectroscopic Analysis. According to the method of Chen and Han [15], the structural change of fermented chicken breast meat was measured using a Jobin Yvon Labram HR800 spectrometer (HORIBA Jobin Yvon Inc., Longjumeau, France). Normalization of individual band intensities was carried out using the phenylalanine amino acid band located near $1003 \mathrm{~cm}^{-1}$ as an internal standard [16]. Protein secondary structures were determined as percentages of $\alpha$-helix, $\beta$-sheet, $\beta$-turn, and random coil according to the method of a previous study [17].

2.6. Low-Field Nuclear Magnetic Resonance (LF-NMR) Measurements. An NMR analyzer (MicroMR20-025V, Niumag Electric Corporation, Shanghai, China) was used to perform LF-NMR measurements. Approximately, $2 \mathrm{~g}$ of sample was placed into NMR glass tubes $(15 \mathrm{~mm}$ in diameter). Proton transverse relaxation times $\left(T_{2}\right)$ were measured using Carr-Purcell-Meiboom-Gill (CPMG) sequence. The analyzer was operated with a resonance frequency of $22.6 \mathrm{MHz}$ at $32^{\circ} \mathrm{C}$, and $\tau$ value was $150 \mu \mathrm{s}$. Data from 10,000 echoes were acquired as 32 scan repetitions. The repetition time between subsequent scans was $8,000 \mathrm{~ms}$. Three relaxation times $\left(T_{2 \mathrm{~b}}, T_{21}\right.$, and $\left.T_{22}\right)$ and their corresponding water populations $\left(P_{2 \mathrm{~b}}, P_{21}\right.$, and $\left.P_{22}\right)$ were recorded. Each measurement was performed at least in triplicate.

\subsection{Analysis of Volatile Flavor Compounds by SPME/GC-MS.} An SPME-fibre $(75 \mu \mathrm{m}$, carboxen/polydimethylsiloxane) was used to extract the volatile compounds. About $4 \mathrm{~g}$ of sample was placed in a $20 \mathrm{~mL}$ headspace transparent glass 
vial. $5 \mu \mathrm{L}$ of 1,2 -dichlorobenzene $(0.1 \mu \mathrm{g} / \mu \mathrm{L}$ in methanol) was added into the samples as an internal standard for quantitative analysis. The SPME-fibre was inserted into the headspace of the glass vial, and the vial was placed at $50^{\circ} \mathrm{C}$ for $30 \mathrm{~min}$ at $800 \mathrm{r} \min ^{-1}$ on a magnetic stirrer (IKA Inc., Staufen, Germany). The SPME-fibre was then desorbed into the GC-MS injector at $250^{\circ} \mathrm{C}$ for $5 \mathrm{~min}$.

A 7890A GC system (Agilent Technologies Co. Ltd., Santa Clara, CA, United States) equipped with a 5975C mass selective detector (Agilent Technologies Co. Ltd., Santa Clara, CA, United States) was used to analyze volatile compounds, which was separated on a fused silica capillary Agilent HP-5 (5\% phenylmethyl siloxane) column $(30 \mathrm{~m} \times 0.25 \mathrm{~mm} \times 0.25 \mu \mathrm{m}$, Agilent Technologies Co. Ltd., Santa Clara, CA, United States). The analysis conditions were in line with the previous study [14].

2.8. Statistical Analyses. All statistical analyses were performed with SPSS 19.0 software package (SPSS Inc., Chicago, IL, USA). One way analysis of variance (ANOVA) with Duncan's test was conducted on the data. Comparisons with $P$ value lower than 0.05 were considered statistically significant.

\section{Results and Discussion}

3.1. Effect of Fermentation on Hardness, Springiness, and Gumminess of Chicken Meat during Fermentation Process. The hardness is an indicator of maturation degree since it is involved in the proteolysis or denaturation of meat proteins and water loss $[18,19]$. As shown in Figure 1, the hardness and springiness of chicken meat remained stable during primary fermentation process but dramatically declined during postfermentation process. The hardness of chicken meat fermented with $P$. nalgiovense and $P$. chrysogenum was decreased by $62.9 \%$ and $53.4 \%$, respectively, compared with unfermented meat (Figure 1(a)). This phenomenon might be attributed to the breakdown of chicken meat proteins during postfermentation, thus generated a great deal of small, lowmolecular weight peptides and damaged the gel network of proteins, consequently reducing firmness of chicken meat samples [20]. It has been reported that gumminess of Turkish dry-fermented sausage gradually showed a significant enhancement during ripening [21]. Similar results also observed in the present study. The gumminess of chicken meat was gradually increased during postfermentation process (Figure 1(c)). An increase of 5.13- and 4.44-fold was found in gumminess after fermentation with $P$. nalgiovense and $P$. chrysogenum, respectively. On the other hand, no significant difference was observed in springiness between $P$. nalgiovense- and P. chrysogenum-fermented chicken meat (Figure 1(b)). However, fermentation with $P$. nalgiovense led to lower hardness and higher gumminess than fermentation with $P$. chrysogenum.

3.2. Effect of Fermentation with Two Molds on Microstructures of Chicken Meat. The SEM and TEM photomicrographs of fermented chicken meat are presented in Figures 2 and 3, respectively. It has been reported that protein degradation might affect the texture of fermented meat [22]. In the present study, surface structures of fermented chicken meat with two molds were similar. They were rough, granular, and adhered together. Hence, the granule formation of chicken meat might be resulted from the myofibril fragment degradation by protease generated from $P$. nalgiovense and $P$. chrysogenum. The decreased hardness and springiness (Figures 1(a) and 1(b)) also further confirmed the protein degradation of chicken meat fermented with $P$. nalgiovense and $P$. chrysogenum. The TEM micrographs of fermented chicken meat with two molds suggested that $Z$ lines of myofibril disappeared and sarcomere fractured and loosely distributed. The boundary of sarcomere was indistinct. A previous study found that high-pressure treatment destroyed myofibrils, $Z$ lines, and $I$ bands of cured Turkey breast; meanwhile, dense aggregation substances formed near the $Z$ line and $M$ line [23].

\subsection{Effect of Fermentation with Two Molds on Protein Sec-} ondary Structure of Chicken Meat. The Raman spectra of fermented chicken meat in $1600-1700 \mathrm{~cm}^{-1}$ range suggested that the most prominent band, centered near around $1653 \mathrm{~cm}^{-1}$ (Figure 4), was assigned to the amide I vibrational mode [15], which consist of amide carbonyl $\mathrm{C}=\mathrm{O}$ stretching vibrations and, to a lesser extent, $\mathrm{C}-\mathrm{N}$ stretching, Ca-C-N bending, and $\mathrm{N}-\mathrm{H}$ in-plane bending of peptide groups $[17,24]$. It has been reported that there is a correlation between the frequencies of the amide $I$ band components and the types of protein backbone conformation [15, 17]. The result of Figure 4 shows that no obvious change was observed in the frequency of the amide I band between chicken meat fermented with $P$. nalgiovense and $P$. chrysogenum. Generally, an amide $I$ band consists of overlapped bands at 1650-1658, 1665-1680, and $1660-1665 \mathrm{~cm}^{-1}$ corresponded to a-helix, $\beta$-sheet, and random coil structure, respectively [16]. In the present study, fermentation with $P$. chrysogenum resulted in higher a-helix percentage than fermentation with $P$. nalgiovense (Figure 5), but the highest percentage of secondary structure was ahelix. Hence, the maximum frequency of the amide $I$ was around $1653 \mathrm{~cm}^{-1}$ (Figure 4).

\subsection{Effect of Fermentation with Two Molds on Protein Tertiary} Structure of Chicken Meat. It has been indicated that several Raman bands are characteristic of the tertiary structure of proteins [16]. Hydrophobic interactions of proteins can be reflected by changes of these Raman bands [16]. The band assigned to tryptophan residues near $760 \mathrm{~cm}^{-1}$ indicates changes in the local environment of the tryptophan aromatic moiety. It has been reported that a decrease in the intensity of a band neat $760 \mathrm{~cm}^{-1}$ may be found when tryptophan residues from a buried, hydrophobic microenvironment become exposed to the polar aqueous solvent [15]. In the present study, no significant difference was observed in the intensity of the Trp band between fermentation with $P$. nalgiovense and $P$. chrysogenum (Table 1). 


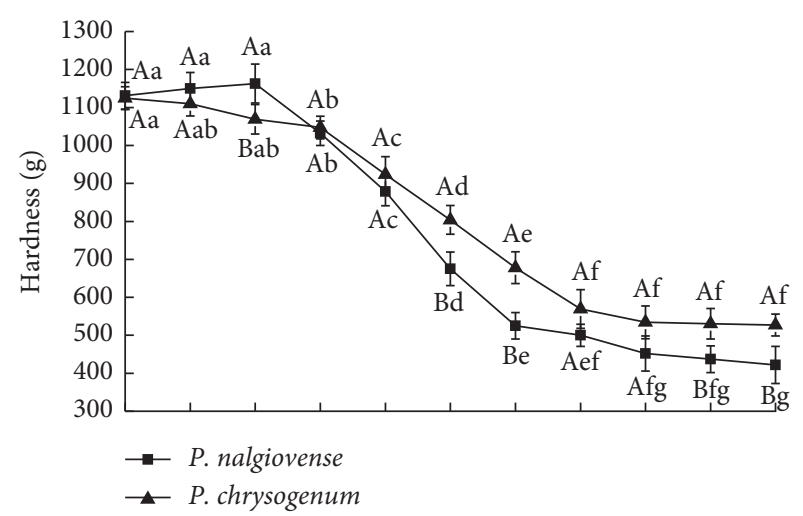

(a)

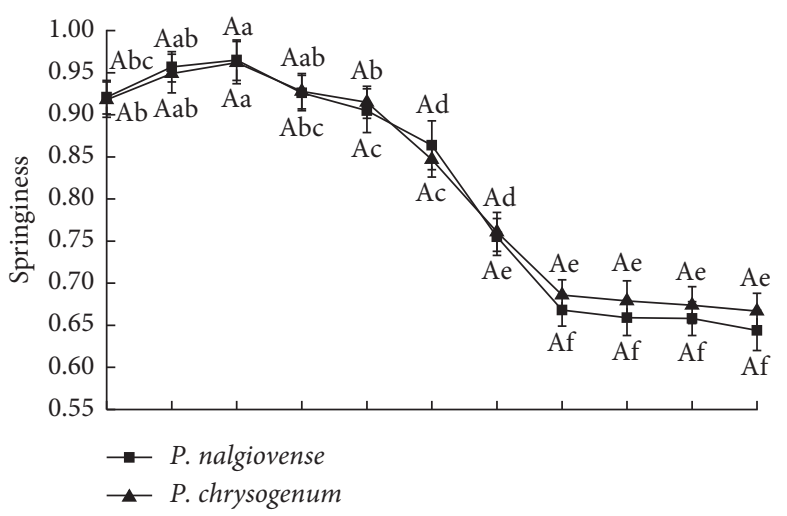

(b)

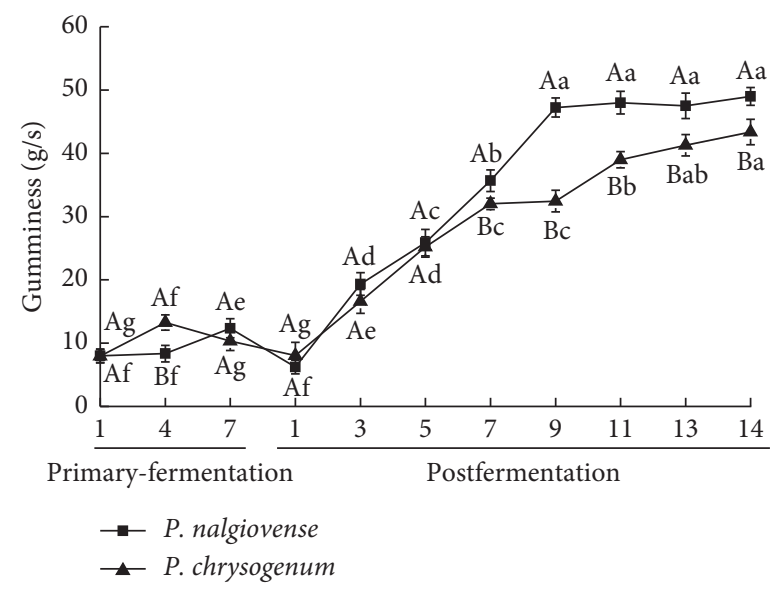

(c)

Figure 1: Effect of fermentation with two molds on hardness (a), springiness (b), and gumminess (c) of chicken meat. The different lowercase and uppercase letters indicate statistical differences for fermentation time and treatments at $p<0.05$, respectively. Samples from ten pieces of chicken breast meat for each treatment were examined.

The doublet bands located near 830 and $850 \mathrm{~cm}^{-1}$ can be used to monitor the microenvironment around tyrosyl residues [25]. The tyrosyl doublet ratio $\left(I_{850} / I_{830}\right)$ has been used as a way of determining whether the tyrosine (Tyr) residue is exposed or buried [17]. The intensity ratio of the doublet bands $\left(I_{850} / I_{830}\right)$ usually ranges from 0.90 to 1.45 [25]. In the present study, the values of the tyrosyl doublet ratio of $I_{850} / I_{830}$ were in this range. Moreover, the band at $850 \mathrm{~cm}^{-1}$ of chicken meat fermented with $P$. nalgiovense and $P$. chrysogenum was more intense than the band at $830 \mathrm{~cm}^{-1}$ (Table 1), indicating that tyrosine residues were buried within the protein network and act as hydrogen donors [25].

3.5. Low-Field NMR. Low-field NMR $T_{2}$ measurements can provide information about the state of the water in samples. The $T_{2 \mathrm{~b}}, T_{21}$, and $T_{22}$ represent bound water, immobilised water, and free water, respectively [26]. Bound water is tightly combined with protein and macromolecular constituents. On the other hand, immobilised water is a component and considered to be located in the myofibrillar network and the protein structure. Additionally, free water is corresponded to water outside the myofibrillar lattice [27]. Figure 6 shows the changes of $T_{2}$ relaxation times of fermented chicken meat. The proportion of three kinds of water in chicken meat is shown in Table 2. In the present study, fermentation with $P$. nalgiovense resulted in higher $T_{2 \mathrm{~b}}$ and $P_{2 \mathrm{~b}}$ than $P$. chrysogenum. The reason might be that fermentation with $P$. nalgiovense caused more muscle myofibrils breakdown and protein denaturation than fermentation with $P$. chrysogenum; meanwhile, the water tightly associated to decreased protein and macromolecular constituents [27]. However, there was no significant difference in immobilised water and free water between fermentation with $P$. nalgiovense and $P$. chrysogenum.

3.6. Effect of Fermentation with Two Molds on Profiles of Volatile Flavor Compounds. The profiles of total volatile compounds in meat could be increased by many factors, such as aging, storage, fermentation, and cooking method. $[28,29]$. On the other hand, volatile acids, esters, and alcohols were the dominant volatiles in the raw chicken breast meat [30]. A large number of alkanes and aldehydes were formed in chicken meat after fermentation with $P$. roquefort $i$ [14]. As shown in Table 3, 45 and 42 kinds of volatile flavor compounds were detected in chicken meat fermented with $P$. nalgiovense and $P$. chrysogenum, respectively. The most 


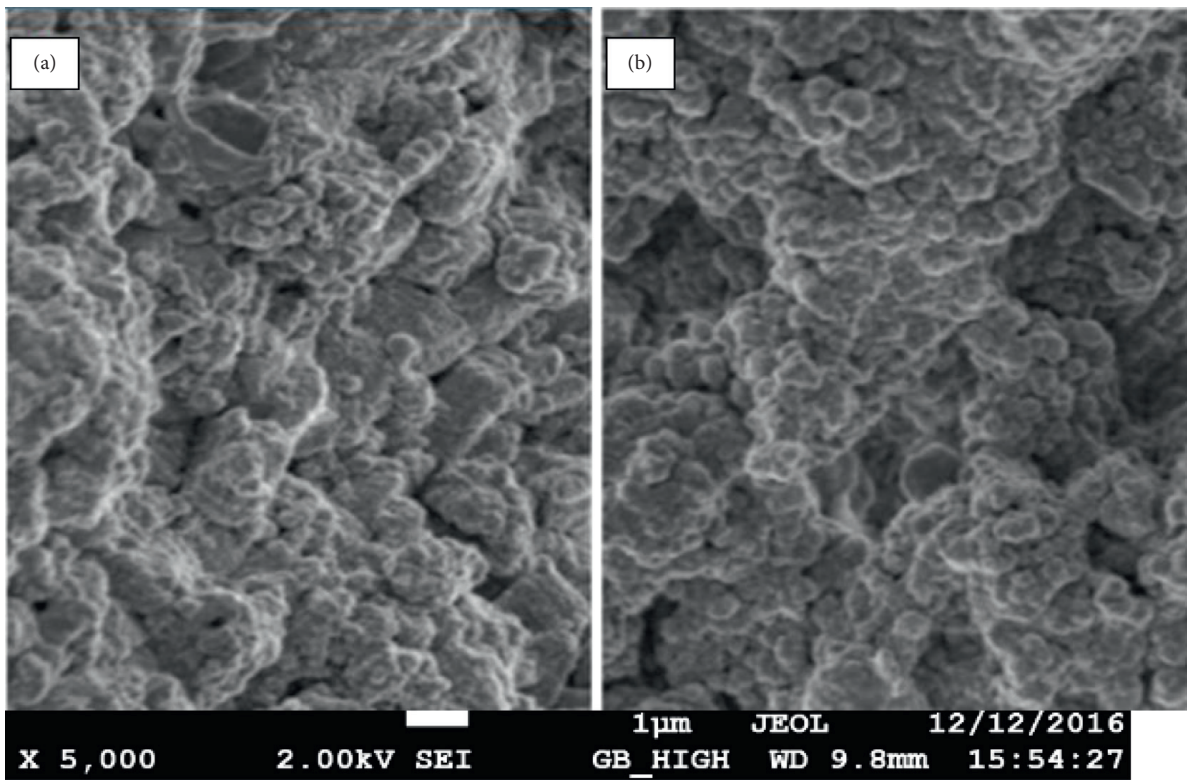

Figure 2: Microstructures of chicken meat fermented with P. nalgiovense (a) and P. chrysogenum (b) observed with SEM. Samples from ten pieces of chicken meat for each treatment were examined.

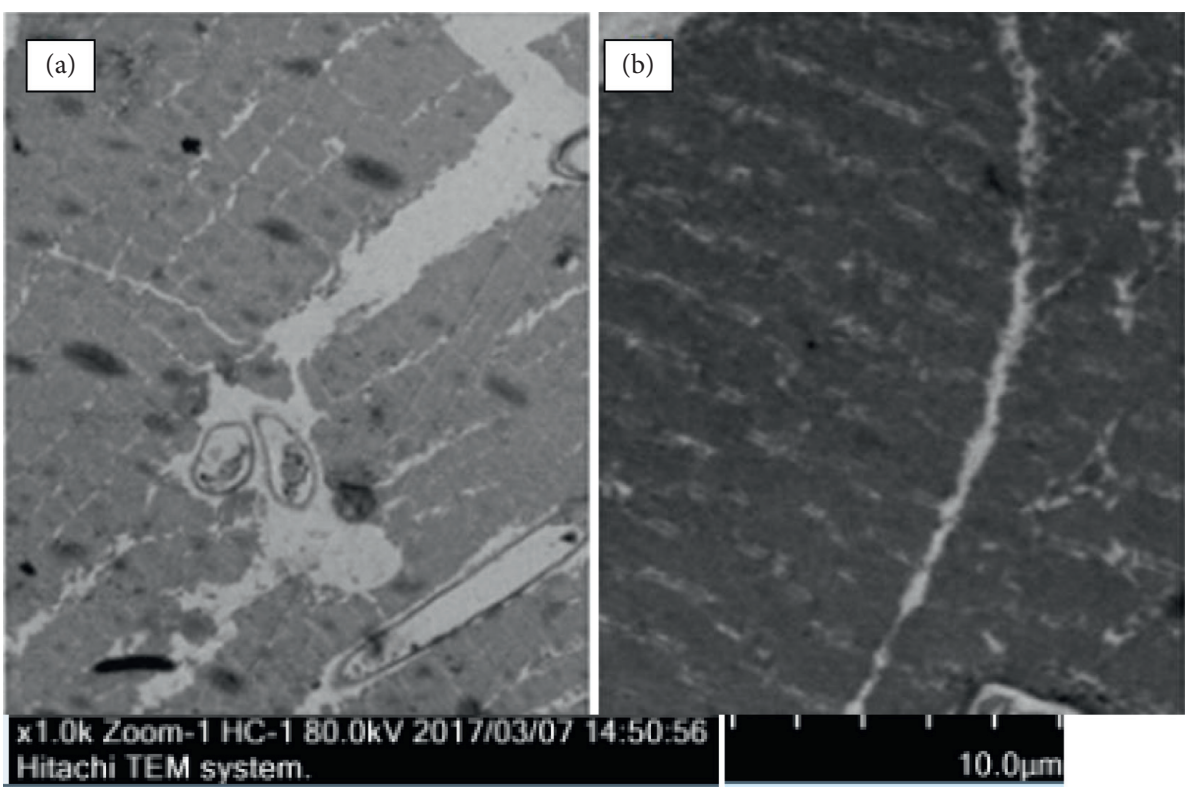

Figure 3: Microstructures of chicken meat fermented with P. nalgiovense (a) and P. chrysogenum (b) observed with TEM. Samples from ten pieces of chicken meat for each treatment were examined.

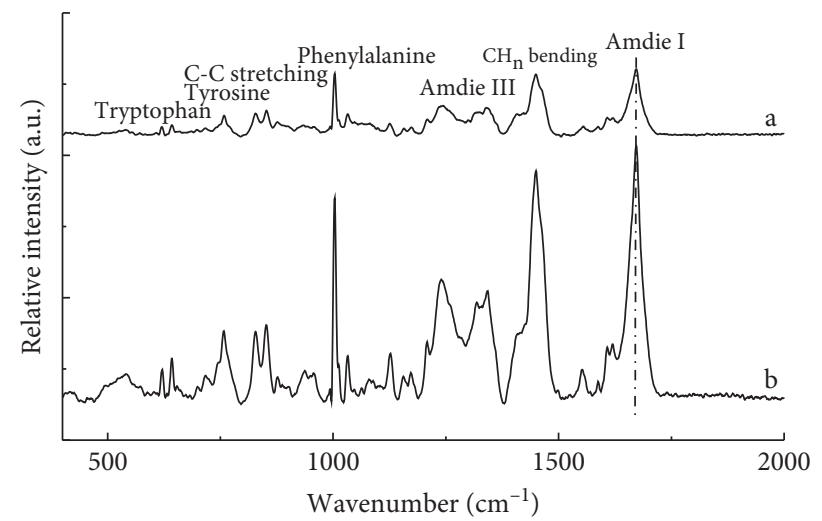

FIGURE 4: Raman spectrum $\left(400-2000 \mathrm{~cm}^{-1}\right)$ of proteins in samples fermented with P. nalgiovense (a) and P. chrysogenum (b). 


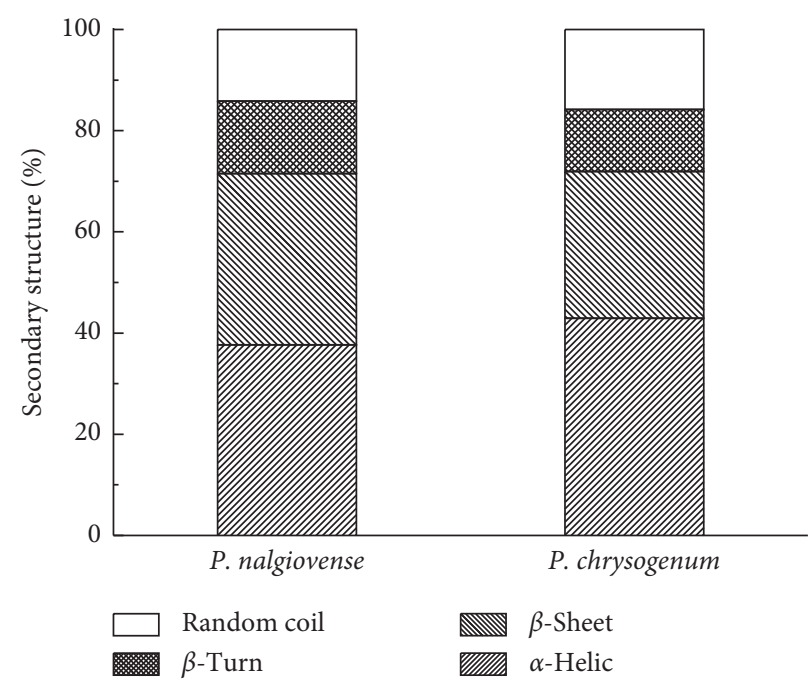

FIGURE 5: Secondary structure fractions estimated from the amide I band in fermented chicken meat with P. nalgiovense and P. chrysogenum.

TABLE 1: Normalized intensities of tryptophan and tyrosine residue microenvironment in fermented chicken meat products.

\begin{tabular}{lcc}
\hline Treatment & Trp band $\left(I_{760} / I_{1003} \mathrm{~cm}^{-1}\right)$ & Tyr doublet $\left(I_{850} / I_{830} \mathrm{~cm}^{-1}\right)$ \\
\hline P. nalgiovense & $0.340 \pm 0.016^{\mathrm{a}}$ & $0.973 \pm 0.014^{\mathrm{a}}$ \\
$P$. chrysogenum & $0.333 \pm 0.030^{\mathrm{a}}$ & $0.906 \pm 0.012^{\mathrm{b}}$ \\
\hline Values not sharing the same letters at the same column are significantly different at $p<0.05$. Samples from ten pieces of chicken breast meat for each
\end{tabular}
treatment were examined.

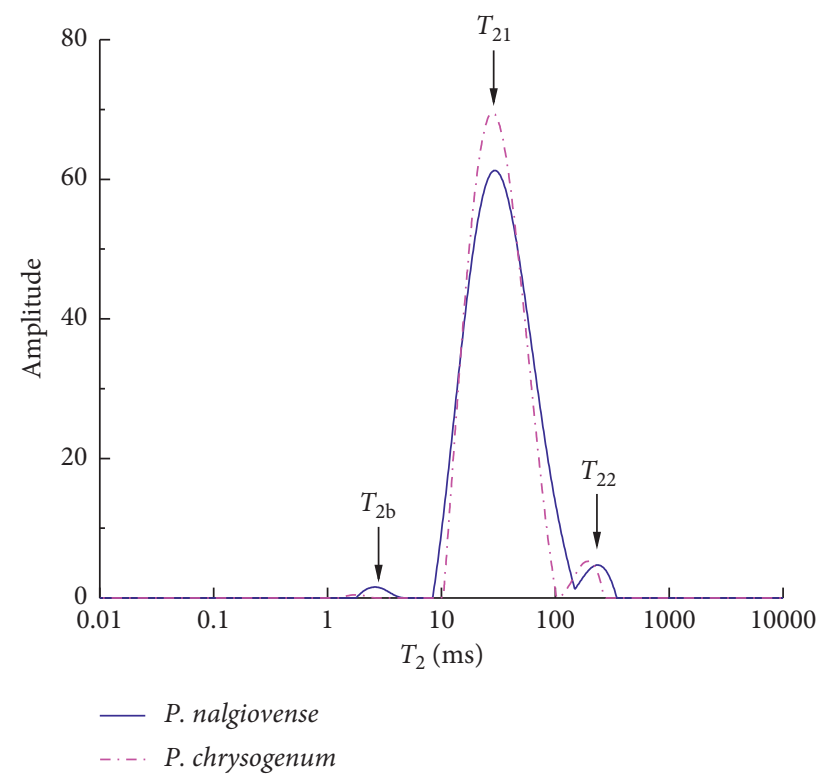

FIgURE 6: Distribution of T2 in chicken meat fermented with P. nalgiovense and P. chrysogenum.

TABLE 2: Water populations of three components (b) in fermented chicken meat.

\begin{tabular}{lcccr}
\hline Processing mode & \multicolumn{2}{c}{$T_{2}$ relaxation time area distribution (\%) } & Total integral areas \\
\hline$P$. nalgiovense & $P_{2 \mathrm{~b}}$ & $P_{21}$ & $P_{22}$ & $7375.17 \pm 44.82^{\mathrm{a}}$ \\
$P$. chrysogenum & $0.80 \pm 0.05^{\mathrm{a}}$ & $96.4 \pm 5.11^{\mathrm{a}}$ & $2.7 \pm 0.97^{\mathrm{a}}$ & $6958.52 \pm 49.01^{\mathrm{b}}$ \\
\hline
\end{tabular}

$P_{2 \mathrm{~b}}, P_{21}$, and $P_{22}$ represent proportion for water closely associated with macromolecules, water located in the myofibrillar network, and extramyofibrillar water, respectively. The different letters indicate statistical differences for the same component at $p<0.05$. Samples from ten pieces of chicken breast meat for each treatment were examined. 
TABLE 3: Effect of fermentation with P. nalgiovense and P. chrysogenum on volatile flavor compounds in chicken meats.

\begin{tabular}{|c|c|c|c|}
\hline \multirow{2}{*}{ Time (min) } & \multirow{2}{*}{ Flavor compound } & \multicolumn{2}{|c|}{ Relative content $(\%)$} \\
\hline & & P. nalgiovense & P. chrysogenum \\
\hline 4.977 & 3-Methylbutyral & 1.276 & 0.783 \\
\hline 6.260 & Toluene & 0.969 & ND \\
\hline 6.706 & n-Dodecane & 1.183 & 0.573 \\
\hline 6.759 & n-Tridecane & 3.908 & 2.735 \\
\hline 7.011 & 2-Methyldecane & 3.486 & 4.869 \\
\hline 7.173 & 10-Methylhendecane & 0.657 & 0.030 \\
\hline 7.258 & Caproaldehyde & 4.209 & 5.032 \\
\hline 7.426 & 3-Methylundecane & 1.013 & 3.081 \\
\hline 7.603 & 2,5-Dimethyl-undecane & 4.206 & 3.092 \\
\hline 7.802 & 2-Methyldodecane & 4.232 & 3.078 \\
\hline 7.879 & n-Tetradecane & 1.455 & 3.982 \\
\hline 7.998 & 3,4,5,6-Tetramethyl octane & 1.109 & 2.553 \\
\hline 8.119 & 2,6-Dimethyldecane & 0.300 & 0.332 \\
\hline 8.219 & 8-Methylhendecane & 5.274 & 5.578 \\
\hline 8.294 & 2,5,6-Trimethyl-decane & 2.345 & 4.982 \\
\hline 8.384 & 6-Methylhendecane & 1.665 & 2.035 \\
\hline 8.804 & 4-Ethyloctane & 2.090 & 6.742 \\
\hline 8.932 & 4-Methylhendecane & 3.366 & 3.254 \\
\hline 9.011 & 2-Methylhendecane & 7.794 & 3.442 \\
\hline 9.146 & 4-Methyldodecane & 6.093 & 10.786 \\
\hline 9.269 & Sulfurous acid-caprate & 5.093 & ND \\
\hline 9.812 & Heptaldehyde & 1.350 & 1.002 \\
\hline 10.147 & Phenylacetic acid ethyl ester & 0.826 & 1.534 \\
\hline 10.316 & n-Pentadecane & 1.383 & 0.606 \\
\hline 10.528 & 3-Methyl-1-butanol & 0.277 & 0.443 \\
\hline 11.051 & 4-Ethyl-2-undecenal & 0.268 & 0.032 \\
\hline 11.244 & 3,7-Dimethyl-1-octanol & 0.384 & 0.069 \\
\hline 12.267 & 3-Hydroxy-2-butanone & 6.351 & 4.058 \\
\hline 12.372 & 1-Octanal & 0.783 & 0.690 \\
\hline 13.175 & trans-2-Heptenal & ND & 0.004 \\
\hline 13.481 & 6-Methyl-5-hepten-2-one & 2.054 & 0.895 \\
\hline 13.900 & 1-Caprylalcohol & 0.166 & 0.698 \\
\hline 14.655 & 1-Nonanal & 1.958 & 0.235 \\
\hline 14.876 & cis-6-Nonenal & 0.017 & 0.345 \\
\hline 15.302 & trans-2-Octenal & 0.207 & 0.743 \\
\hline 15.571 & Acetate acid & 12.820 & 14.541 \\
\hline 16.464 & 1-Decylalcohol & 0.057 & 2.099 \\
\hline 16.478 & 1-Tetradecanol & 0.296 & 0.961 \\
\hline 16.844 & Benzaldehyde & 0.259 & 0.343 \\
\hline 17.069 & trans-2-Nonenal & 0.188 & ND \\
\hline 17.272 & 2-Pyridinecarboxaldehyde & 1.356 & 0.112 \\
\hline 18.212 & 2,3-Butanediol & 0.647 & ND \\
\hline 18.351 & Butyric acid & 2.085 & 0.798 \\
\hline 19.961 & 2-Undecenal & 0.973 & 0.542 \\
\hline 20.649 & trans-2,4-Decadienial & 0.658 & 0.092 \\
\hline 21.027 & Hexanoic acid & 1.054 & 0.075 \\
\hline
\end{tabular}

ND represents not detectable.

abundant volatile compound in $P$. nalgiovense- and $P$. chrysogenum-fermented chicken meat was alkanes, which accounted for $52.5 \%$ and $63.1 \%$, respectively. Song et al. [31] reported that the enhancement of aldehydes was resulted from the decomposition of oleic acid released as a result of lipolysis by lipase. Likewise, 11 kinds of aldehydes were identified in $P$. nalgiovense- and $P$. chrysogenum-fermented chicken meat in the present study, and they were accounted for $11.5 \%$ and $9.8 \%$ of the total volatiles, respectively.

In addition, ketones and alcohols can be formed by the lipid degradation [31, 32]. Particularly, alcohols are formed due to the oxidation of the fatty acid components, which are derived from lipid degradation [32]. In the present study, only two ketones, 6-methyl-5-hepten-2one and 3-hydroxy-2-butanone, were identified in fermented samples, and the content in samples fermented with $P$. nalgiovense was higher than that in samples fermented with $P$. chrysogenum (Table 3). Mehmet et al. [30] showed that alcohols play a very small role in odour because they have relatively high odour threshold. In $P$. nalgiovense- and P. chrysogenum-fermented chicken meat, 6 and 5 kinds of alcohols were detected, 
respectively. However, their relative content was lower than alkanes and aldehydes.

\section{Conclusion}

The textural properties, structure, water distribution, and volatile compounds were changed by fermentation with $P$. nalgiovense and $P$. chrysogenum. Lower hardness and higher gumminess were observed in $P$. nalgiovense-fermented chicken meat. Meanwhile, granule formed and sarcomere fractured, which were observed by SEM and TEM analysis. Samples fermented with $P$. chrysogenum contained higher $\alpha$-helix percentage than those fermented with $P$. nalgiovense. However, tyrosine residues were buried within the protein network after fermentation with $P$. nalgiovense and $P$. chrysogenum. Higher bound water proportion was observed in P. nalgiovense-fermented samples. The predominant volatile compounds in fermented samples with two molds were alkanes, subsequently aldehydes. These results indicated that fermentation with the two molds could be useful ways to improve quality of chicken meat. Some experiments will be conducted to evaluate the effect of the mixed molds used as starter cultures on chicken and duck meat. The present study provides valuable information for the development of chicken product and industry in China.

\section{Data Availability}

The data used to support the findings of this study are included within the article.

\section{Conflicts of Interest}

The authors declare that they have no conflicts of interest.

\section{Acknowledgments}

This work was supported by the financial support provided by the Shandong Modern Agricultural Technology and Industry System (SDAIT-11-11), China Agriculture Research System (CARS-41-Z06), and National Key R\&D Projects (2018YFD0501400). This work also supported by the National Natural Science Foundation of China (no. 31501512).

\section{References}

[1] L. O. Sunesen and L. H. Stahnke, "Mould starter cultures for dry sausages-selection, application and effects," Meat Science, vol. 65, no. 3, pp. 935-948, 2003.

[2] J. M. Bruna, E. M. Hierro, L. de la Hoz, D. S. Mottram, and M. Fernandez, "Changes in selected biochemical and sensory parameters as affected by the superficial inoculation of Penicillium camemberti on dry fermented sausages," International Journal of Food Microbiology, vol. 85, no. 1, pp. 111-125, 2003.

[3] V. Bernáldez, J. J. Córdoba, M. Rodríguez, M. Cordero, L. Polo, and A. Rodríguez, "Effect of Penicillium nalgiovense as protective culture in processing of dry-fermented sausage "salchichón"” Food Control, vol. 32, no. 1, pp. 69-76, 2013.

[4] D. T. Asefa, R. O. Gjerde, M. S. Sidhu et al., "Moulds contaminants on Norwegian dry-cured meat products,"
International Journal of Food Microbiology, vol. 128, no. 3, pp. 435-439, 2009.

[5] J. M. Bruna, E. M. Hierro, L. de la Hoz, D. S. Mottram, M. Fernández, and J. A. Ordóñez, "The contribution of Penicillium aurantiogriseum to the volatile composition and sensory quality of dry fermented sausages," Meat Science, vol. 59, no. 1, pp. 97-107, 2001.

[6] A. Martín, J. J. Córdoba, F. Núñez, M. J. Benito, and M. A. Asensio, "Contribution of a selected fungal population to proteolysis on dry-cured ham," International Journal of Food Microbiology, vol. 94, pp. 55-66, 2004.

[7] A. Martin, J. Córdoba, E. Aranda, M. Cordoba, and M. Asensio, "Contribution of a selected fungal population to the volatile compounds on dry-cured ham," International Journal of Food Microbiology, vol. 110, no. 1, pp. 8-18, 2006.

[8] M. Papagianni and E. M. Papamichael, "Modeling growth, substrate consumption and product formation of Penicillium nalgiovense grown on meat simulation medium in submerged batch culture," Journal of Industrial Microbiology \& Biotechnology, vol. 34, no. 3, pp. 225-231, 2006.

[9] J. E. Kinsella, D. H. Hwang, and B. Dwivedi, "Enzymes of penicillium roqueforti involved in the biosynthesis of cheese flavor," C R C Critical Reviews in Food Science and Nutrition, vol. 8, no. 2, pp. 191-228, 1976.

[10] F. Alisson-Silva, K. Kawanishi, and A. Varki, "Human risk of diseases associated with red meat intake: analysis of current theories and proposed role for metabolic incorporation of a non-human sialic acid," Molecular Aspects of Medicine, vol. 51, pp. 16-30, 2016.

[11] A. Wolk, "Potential health hazards of eating red meat," Journal of Internal Medicine, vol. 281, no. 2, pp. 106-122, 2017.

[12] M. Cosgrove, A. Flynn, and M. Kiely, "Consumption of red meat, white meat and processed meat in Irish adults in relation to dietary quality," British Journal of Nutrition, vol. 93, no. 6, pp. 933-942, 2005.

[13] S. D. Da, A. M. V. de Arruda, and A. A. Gonçalves, "Quality characteristics of broiler chicken meat from free-range and industrial poultry system for the consumers," Journal of Food Science and Technology, vol. 54, pp. 1818-1826, 2017.

[14] L. Guo, B. Yu, S. Wang et al., "Effect of ripening with Penicillium roqueforti on texture, microstructure, water distribution and volatiles of chicken breast meat," International Journal of Food Science \& Technology, vol. 54, pp. 1818-1826, 2019.

[15] H. Chen and M. Han, "Raman spectroscopic study of the effects of microbial transglutaminase on heat-induced gelation of pork myofibrillar proteins and its relationship with textural characteristics," Food Research International, vol. 44, no. 5, pp. 1514-1520, 2011.

[16] A. M. Herrero, M. I. Cambero, J. A. Ordóñez, L. de la Hoz, and P. Carmona, "Raman spectroscopy study of the structural effect of microbial transglutaminase on meat systems and its relationship with textural characteristics," Food Chemistry, vol. 109, no. 1, pp. 25-32, 2008.

[17] P. Tadpitchayangkoon, J. W. Park, S. G. Mayer, and J. Yongsawatdigul, "Structural changes and dynamic rheological properties of sarcoplasmic proteins subjected to $\mathrm{pH}$ shift method," Journal of Agricultural and Food Chemistry, vol. 58, no. 7, pp. 4241-4249, 2010.

[18] M. Laranjo, A. C. Agulheiro-Santos, M. E. Potes et al., "Effects of genotype, salt content and calibre on quality of traditional dry-fermented sausages," Food Control, vol. 56, pp. 119-127, 2015. 
[19] Y. Wu, M. Zhao, B. Yang, W. Sun, C. Cui, and L. Mu, " "Microbial analysis and textural properties of Cantonese sausage," Journal of Food Process Engineering, vol. 33, no. 1, pp. 2-14, 2010.

[20] S. Maqsood, K. Manheem, A. Gani, and A. Abushelaibi, "Degradation of myofibrillar, sarcoplasmic and connective tissue proteins by plant proteolytic enzymes and their impact on camel meat tenderness," Journal of Food Science and Technology, vol. 55, 2018.

[21] M. Kargozari, S. Moini, A. Akhondzadeh Basti et al., "Development of Turkish dry-fermented sausage (sucuk) reformulated with camel meat and hump fat and evaluation of physicochemical, textural, fatty acid and volatile compound profiles during ripening," LWT-Food Science and Technology, vol. 59, no. 2, pp. 849-858, 2014.

[22] S. Fadda, M. J. Vildoza, and G. Vignolo, "The acidogenic metabolism of lactobacillus plantarum crl 681 improves sarcoplasmic protein hydrolysis during meat fermentation," Journal of Muscle Foods, vol. 21, no. 3, pp. 545-556, 2010.

[23] M. F. Villacís, N. K. Rastogi, and V. M. Balasubramaniam, "Effect of high pressure on moisture and $\mathrm{NaCl}$ diffusion into Turkey breast," LWT-Food Science and Technology, vol. 41, no. 5, pp. 836-844, 2008.

[24] A. M. Herrero, P. Carmona, S. Cofrades, and F. JiménezColmenero, "Raman spectroscopic determination of structural changes in meat batters upon soy protein addition and heat treatment," Food Research International, vol. 41, no. 7, pp. 765-772, 2008.

[25] J.-H. Shao, Y.-F. Zou, X.-L. Xu, J.-Q. Wu, and G.-H. Zhou, "Evaluation of structural changes in raw and heated meat batters prepared with different lipids using Raman spectroscopy," Food Research International, vol. 44, no. 9, pp. 2955-2961, 2011.

[26] J.-H. Shao, Y.-M. Deng, N. Jia et al., "Low-field NMR determination of water distribution in meat batters with $\mathrm{NaCl}$ and polyphosphate addition," Food Chemistry, vol. 200, pp. 308-314, 2016.

[27] Z.-L. Kang, B. Li, H.-J. Ma, and F.-S. Chen, "Effect of different processing methods and salt content on the physicochemical and rheological properties of meat batters," International Journal of Food Properties, vol. 19, no. 7, pp. 1604-1615, 2015.

[28] R. Domínguez, M. Gómez, S. Fonseca, and J. M. Lorenzo, "Effect of different cooking methods on lipid oxidation and formation of volatile compounds in foal meat," Meat Science, vol. 97, no. 2, pp. 223-230, 2014.

[29] T. A. Misharina, V. A. Andreenkov, and E. A. Vashchuk, "Changes in the composition of volatile compounds during aging of dry-cured sausages," Applied Biochemistry and Microbiology, vol. 37, no. 4, pp. 413-418, 2001.

[30] T. A. Mehmet, F. Gokhan, and S. Serkan, "Evaluation of volatile compounds in chicken breast meat using simultaneous distillation and extract," Journal of Food and Nutrition Research, vol. 53, pp. 137-142, 2014.

[31] S. Song, Q. Tang, L. Fan et al., "Identification of pork flavour precursors from enzyme-treated lard using Maillard model system assessed by GC-MS and partial least squares regression," Meat Science, vol. 124, pp. 15-24, 2017.

[32] D. D. Jayasena, D. U. Ahn, K. C. Nam, and C. Jo, "Flavour chemistry of chicken meat: a review," Asian-Australasian Journal of Animal Sciences, vol. 26, no. 5, pp. 732-742, 2013. 